\title{
Moderate intensity exercise inhibits macrophage infiltration and attenuates adipocyte inflammation in ovariectomized rats
}

\author{
Jong Kui Jun', Wang Lok Lee', Hee Geun Park, Sang Ki Lee ${ }^{1}$, Sun Hyo Jeong ${ }^{2}$ and Young Ran Lee ${ }^{1 *}$ \\ ${ }^{1}$ Department of Sports Science, Chungnam National University, Daejeon, Korea \\ ${ }^{2}$ Department of Science Management, Mokwon University, Daejeon, Korea
}

(Received: 2014/02/03, Revised: 2014/02/18, Published online: 2014/02/27)

[Purpose] The purpose of this study was to investigate the effects of the different endurance exercise intensities on the macrophage infiltration and adipocyte inflammation of ovariectomized rats. [Methods] 24 female SD rats (6 weeks old) were randomly assigned to sham control $(\mathrm{SC} ; \mathrm{n}=6)$, ovariectomized control $(\mathrm{OC} ; \mathrm{n}=6)$, ovariectomized low intensity exercise $(\mathrm{OL} ; \mathrm{n}=6)$, and ovariectomized moderate intensity exercise $(\mathrm{OM} ; \mathrm{n}=6)$ groups. The two training groups ran for $60 \mathrm{~min} /$ day, 5 times/ week at 18 and $26 \mathrm{~m} / \mathrm{min}$ for 16 weeks. Twenty-four hours after the last exercise session, rats were sacrified, and epididymal pads were analyzed. F4/80 and IL-6 expressions were evaluated by western blotting. ICAM-1, VCAM-1 TLR4, TNF- $a$, and MCP-1 mRNA expressions were evaluated by RT-PCR. [Results] In comparison with OC group, OM group showed significantly lower body weight gain and adipose tissue mass. Also, OM group markedly inhibited F4/80 expression, adhesion molecule (ICAM-1, VCAM-1) and pro-inflammatory cytokines (TLR4, TNF-a, MCP-1) mRNA expressions in adipose tissue. In contrast, OL group partially prevented body weight gain while other examined parameter were unaffected by low intensity exercise training. [Conclusion] The results of this study suggest that OM group inhibits visceral macrophage infiltration by suppressing the adhesion molecules. It may also attenuate cytokine production in the adipose tissue by repressing the TLR4-mediated pro-inflammatory signaling cascades in ovariectomized rats. [Keyword] exercise intensity, macrophage, inflammation, ovariectomized

\section{INTRODUCTION}

It has been reported that menopause is closely related to the pathogenesis of the metabolic syndrome such as lipid abnormality, obesity, and type II diabetes mellitus due to the decrease of estrogen concentration [1].

The animals of which ovary is removed (hereafter referred as to ovariectomized animals) have been used as an animal model to be improved obesity and metabolic imbalances that estrogen secretion appears by being limited [2,3]. In fact, the animals which ovarian function is lost show the increased body weight and fat weight, and much insulin resistance, but it has been reported that if their estrogen are treated, these phenomenon are improved [4-7]. In particular, it has been suggested that the reduction of hormones due to the loss of ovarian function is the important factor that allows the visceral obesity to occur while reporting that in the case of the group that the ovaries are removed, the weight of abdominal white adipose (WAT :white adipose tissue) was more significantly increased in comparison with the group of normal person $[8,9]$. However, because many studies [2-7] reported up to now have been run parallel with the high fat dietary along with the removal of ovary, it is considered that cannot exclude the high fat dietary effect in the process improving obesity. Thus, Nicole et al. [10], have reported that the study on ovariectomized animal is important to clearly determine the mechanism of metabolic imbalance induced due to just the reduction of hormone by separating with the obese animals induced by the high fat dietary.

Visceral obesity has been well known as major risk factor of metabolic diseases featuring the chronic low-grade inflammation $[11,12]$. The inflammatory response in adipose tissue is caused when immune cells are migrated to the damaged tissue, which is the expression of the cell adhesion molecule that conducts an important role in the migration process of these cells. Also, it has been reported that the

\footnotetext{
* Corresponding author: Youngran Lee, Tel. 82-42-821-7582, Fax. 82-42-823-0387, Email. yrlee@cnu.ac.kr

(c) 2014 The Korean Society for Exercise Nutrition
} 
expression of cell adhesion molecules, the chemokine that induces chemotaxis, and various inflammation-related receptors are associated complexly [13,14]. Recently, it has been reported that the monocyte infiltrated into adipose tissue is differentiated into macrophages, and it plays an important role in the deterioration of the inflammatory conditions in the adipose tissue by increasing the secretion of the cytokine and chemokines $[9,10]$. Thus, studying the treatment method that suppresses the macrophage infiltrate owing to the loss of ovarian function, or that interrupts the path of inflammatory signal, it may say that the possibility to prevent the metabolic disease is large.

Regular physical activity depending on its intensity and duration induces the change of the function of macrophages and the level of immunity. It has been reported that the function of the mouse peritoneal macrophages with even a one-time moderate intensity exercise is increased $[15,16]$, and long-term swimming exercise increases the phagocytic in the peritoneal macrophage of animal model. In another study, it has been known that the moderate intensity exercise allows to reduce the inflammatory cytokines on adipose tissue of obese patient and animal, and it reduces the expression of F4/80 mRNA used as a marker of macrophage, but it has been reported that the specific mechanisms are not clear $[18,19]$.

In particular, while reporting that the woman in the menopause gives positive change in weight control and body fat amount by the low intensity exercise such as walking at least 30 minutes every day, and while reporting that influences also on blood lipid by activating fat metabolism, and it was suggested that the side effect of regular physical activity is smaller and the tendency of increase of body weight is relieved in comparison with hormone therapy or dietary therapy, and the risk factors of cardiovascular disease can be reduced $[20,21]$.

In another study, Tjonna et al. [22], have reported that as the results that conduct the low-intensity exercise and moderate intensity exercise targeting the patient with metabolic syndrome, both intensity exercises showed equal effect in the weight reduction of body weight and body fat, but the increase of high-density lipoprotein cholesterol and insulin sensitivity appears only in moderate intensity exercise [23], and Drenth et al. [23], have reported that the medium strength exercise reduces the expression of inflammatory cytokines or there was no change, and high-intensity exercise increases rather the expression of the inflammatory cytokines [25]. Thus, the regular exercise is actively recommended as a tool for the prevention of metabolic disease by improving positively the body weight and body fat of an obese, but the conclusions conflicted each other are being drawn depending on the exercise intensity or duration.

Thus, in this study, the authors intended to investigate each exercise effect for the infiltration of macrophages and the inflammatory response in adipose tissue, after conducting low intensity exercise and moderate intensity exercise for 16 weeks by using the ovariectomized animal models.

\section{METHODS}

\section{Animals care}

The experimental animals were used by parceling out 24 female Sprague-Dawley rats of 6 weeks from the Central Lab. Animal Inc. In order to expose to the stress same as the ovariectomized animals, six rats were separated as a control group by performing the sham operation, and the remaining 18 rats had the adjustment period in a laboratory during 1 week after conducting both ovariectomized. The breeding environment of the experimental animal was the condition of the mean temperature of $22 \pm 2^{\circ} \mathrm{C}$, the humidity of $50 \pm$ $5 \%$, and the circulation of day/night was maintained in the cycle of 12 hours (06:00 18:00 daytime), and feed and water were enough supplied until the end time of the experiment. The classification of groups were classified into total four groups such as sham-operated control (SC), Ovariectomized control (OC), Ovariectomized low-intensity exercise (OL) and Ovariectomized moderate-intensity exercise (OM), and six rats per group were assigned. The body weight of each group were measured and recorded once a week, and the handling of the experimental animals received approval by submitting a proposal to Institutional Animal Care and Use Committee (No, CNU-00203), and bred and handled in accordance with the handling regulation of Animal Experimentation Committee in Chungnam National University.

\section{Exercise protocol}

For the exercise type of ovariectomized animals, the aerobic exercise using the treadmill was selected. The animals underwent the treadmill exercise adaptation period (8-10 $\mathrm{m} / \mathrm{min}$ ) during laboratory adjustment period of a week-long after removing their ovaries. After an adaptation period of exercise, the settings of exercise intensity were performed for total 16 weeks after dividing into Low intensity exercise $(\mathrm{OL}$, $n=6)$, and moderate intensity exercise $(\mathrm{OM}, \mathrm{n}=6)$ based on the previous studies [20,21]. The low intensity exercise group (VO2max $\sim 50 \%$ ) was applied at the rate of $18 \mathrm{~m} / \mathrm{min}$, and 
the moderate intensity exercise group ( $\mathrm{VO} 2 \mathrm{max} \sim 70 \%$ ) conducted running exercise of five times per week at the same time for 60 minutes a week at the rate of $26 \mathrm{~m} / \mathrm{min}$. In order to minimize the stress of animals, any external stimuli or electric impact did not apply, and the control group was exposed in the same environmental stress and treadmill noise and vibration as the exercise group, and the supplies of feeds and water were also limited.

\section{$R N A$ extraction}

In order to extract Total RNA, $100 \mu \mathrm{g}$ of epididymal fat tissue was put into Trizol (Qiagen, Hilden, Germany) and the tissue was grinded for 20 seconds using a homogenizer, and the homogenized solution prepared from the homogenizer was allowed to stand at room temperature for 5 minutes. Chloroform (Sigma, USA) of $200 \mu \mathrm{l}$ was added into this solution, and then the solution was allowed to stand for 3 minutes at room temperature after mixing for 15 seconds so that the chloroform was wholly mixed well, and was centrifuged $\left(13,000 \mathrm{rpm}, 4^{\circ} \mathrm{C}, 15 \mathrm{~min}\right.$.). The only clear supernatant solution was separated from the centrifuged solution into a new tube, and then was allowed to stand at room temperature for 10 minutes after adding the same amount of isopropanol (Sigma, USA) into this supernatant solution, and it was centrifuged $\left(13,000 \mathrm{rpm}, 4^{\circ} \mathrm{C}, 10 \mathrm{~min}\right.$.). $70 \%$ ethanol of $1 \mathrm{ml}$ was added on RNA pellet formed on the bottom of tube by centrifugation, and the pellet was washed twice $\left(4,500 \mathrm{rpm}, 4^{\circ} \mathrm{C}, 5 \mathrm{~min}\right.$.). If the RNA pellet was completely dried, the RNA pellet was dissolved by adding the $0.01 \%$ DEPC-treated distilled water of $30 \mu l$. The array of forward and reverse primer is the same as shown in Table 1.

\section{$R T-P C R$}

The quantity of mRNA was measured using Reverse transcription-polymerase chain reaction (RT-PCR). Complementary DNA was heat treated at $75^{\circ} \mathrm{C}$ for 15 minutes by preparing final about $14 \mu \mathrm{l}$ after mixing total RNA of $2 \mu \mathrm{g}$ and reverse primer of $0.5 \mu \mathrm{g}$, and then was stored in ice for 5 minutes. 5X M-MLV reaction buffer, $10 \mathrm{mM}$ dNTP mixture, and 200 units M-MLV RT (Promega, Madison, WI, USA) were added into RNA, and the final amount was allowed to $25 \mu \mathrm{l}$, and then was reacted at $42^{\circ} \mathrm{C}$ for 1 hour. 10X reaction buffer (Mg2 + containing), 10mM dNTP, 5 unites Taq polymerase (Solgent, Taeon, Korea), and $10 \mu \mathrm{M}$ primer were added into the RT reaction of $5 \mu \mathrm{l}$, and this mixing solution was allowed to final $50 \mu \mathrm{l}$, and then PCR was performed using TC-100TM Programmable Thermal Controller (MJ, Research, INC., Waltham, MA, USA).

\section{Western blotting}

The epididymal fat tissue of $0.2 \mathrm{mg}$ was quantified using a microbalance, and crushed by adding the protein extraction solution (Pro-prep, iNtron) of $500 \mu \mathrm{l}$ on it. The supernatant was separated by performing the centrifugation at the speed of $3000 \mathrm{rpm}$ at $4{ }^{\circ} \mathrm{C}$ for 20 minutes, and then the amount of protein was quantified at the absorbance of $550 \mathrm{~nm}$ using BCA assay Kit (Bio-rad, USA).

Protein was separated on $10 \%$ SDS-PAGE and was electro transferred onto nitrocellulose membrane. PVDF membrane (Schleicher \& Schuell Bioscience), Whatman 3M paper, and PVDF membrane (Millipore) soaked with Transfer buffer (190 mM glycine, $50 \mathrm{mM}$ Tris-base, $0.05 \%$ SDS, $20 \%$ methanol) were sequentially overlapped, and then transferred by $50 \mathrm{~V}$ for about 50 minutes by installing on Mini transblot module (Bio Rad). If the deposition on the membrane was completed, the deposited sample allowed blocking by the skim milk solution of $5 \%$ for 2 hours on the rocker platform. After the reaction was conducted, primary antibody F480 (Santa Cruz, USA) and IL-6 (Santa Cruz, USA)are treated and allowed to stand at $4{ }^{\circ} \mathrm{C}$ state for overnight, and then the secondary antibody was shaken for 2 hours by diluting with blocking solution to 1:1000, and then washed 3 times for 10 minutes with TBS-T solution. Finally, the immunoreactive band of protein was confirmed by a film exposure through ECL system using ECL (enhanced chemiluminescence) solution Dura (Thermo, USA). The protein appeared on the

Table 1. Sequences of oligonucleotide primer for RT-PCR

\begin{tabular}{lll}
\hline \multicolumn{1}{c}{ Gene } & \multicolumn{1}{c}{ Forward primer } & Reverse Primer \\
\hline TLR 4 & ATGGCATGGCTTACACCACC & GAGGCCAATTTTGTCTCCACA \\
TNF- $a$ & CAAGGGACAAGGCTGCCCCG & TAGACCTGCCCGGACTCCGC \\
MCP-1 & AGGTCCCTGTCATGCTTCTG & TCTGGACCCATTCCTTCTTG \\
ICAM-1 & CCTGATGGGCAGTCAACAGCTA & ACAGCTGGCTCCCGTTTCA \\
VCAM-1 & CTTCATCCCCACCATTGAAG & TGAGCAGGTCAGGTTCACAG \\
B-actin & TCACCCACACTGTGCCCATCTACGA & CAGCGGAACCGCTCATTGCCAATGG \\
\hline
\end{tabular}


band was quantified by the relative intensity using Scion image (Scion Corp.Frederick, Md., USA) program.

\section{Statistical methods}

For all the data, the average and standard error for the analysis items by each group were calculated using the statistical program of SPSS. One-way ANOVA was performed in order to verify the effect by variables between groups, and LSD was used for the post verification. All statistical significance level was set to $p<.05$.

\section{RESULTS}

\section{Change of body weight and epididymal fat mass}

The change of the body weight of and epididymal fat tissue an ovariectomized animal are the same as in Table 2.

The body weight of OC group and adipose tissue weight were significantly increased in comparison with $\mathrm{SC}$ group $(p$ $<.01)$. After the treatment of 16 weeks, the body weight in OL group and the body weight and adipose tissue weight in OM group were effectively reduced in comparison with OC group $(p<.01)$.

\section{Expression of F480 in ovariectomized rats}

The changes in F480 expression amount of the ovariectomized animals are the same as in Fig. 1.

The F480 expression amount of OC group was significantly increased in comparison with SC group $(p<.01)$. After the exercise treatment of 16 weeks, OM group allowed to effectively suppress F480 in comparison with OC group ( $p$ $<.05)$. However, OL group did not represent the significant difference.

\section{Expression of ICAM-1 and VCAM-1 in ovariectomized rats}

The change of ICAM-1 and VCAM-1 mRNA expression of the ovariectomized animals is the same as in Fig. 2.

The ICAM-1 mRNA expression of OC group was
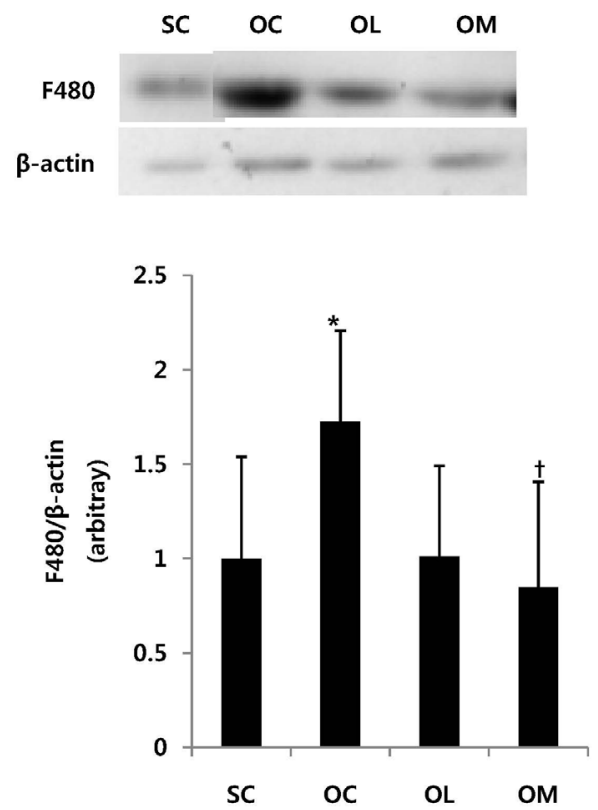

Fig. 1. Expression of F480 mRNA in ovariectomized rats. All values are expressed as means \pm SD. $P$-values are the result of post hoc LSD test when the one-way ANOVA was significant. $* p<.05$ significantly different to the $\mathrm{SC}$ group. ${ }^{\dagger} p<.05$ significantly different to the $\mathrm{OC}$ group.

significantly increased in comparison with $\mathrm{SC}$ group $(p<.01)$. After the exercise treatment of 16 weeks, OM group allowed to effectively reduce ICAM-1 mRNA expression in comparison with $\mathrm{OC}$ group $(p<.01)$. However, OL group did not represent the significant difference. VCAM-1 mRNA expression showed significant increase in $\mathrm{OC}$ and $\mathrm{OL}$ group in comparison with SC group. After the exercise treatment of 16 weeks, OM group allowed to effectively reduce VCAM-1 mRNA expression in comparison with OC group and OL group $(p<.01)$.

Expression of IL-6, TLR4, TNF- a, MCP-1 in ovariectomized rats

The change of IL-6, TLR4, TNF- $a$ and MCP-1 mRNA of the ovariectomized animal is the same as in Fig. 3.

The IL-6 expression of OC group was significantly increased in comparison with SC group $(p<.01)$. After the exercise treatment of 16 weeks, OL group and OM group were not shown the significant difference for IL-6 expression

Table 2. Change of body weight and epididymal fat mass

\begin{tabular}{lcccc}
\hline & SC & OC & OL & OM \\
\hline Body weight $(\mathrm{g})$ & $270.55 \pm 18.19$ & $339.82 \pm 21.86^{*}$ & $335.99 \pm 25.35^{*}$ & $311.47 \pm 27.7 *^{+}$ \\
Epididymal fat $(\mathrm{g})$ & $4.21 \pm 1.36$ & $8.08 \pm 2.53^{*}$ & $6.33 \pm 1.96^{*^{+}}$ & $5.23 \pm 1.32^{+}$ \\
\hline
\end{tabular}

Values are mean \pm SD. SC; Sham operated control, OC; ovariectomized control, OL; ovariectomized low intensity exercise, OM;ovariectomized moderate intensity exercise. ${ }^{*} p<.05$ significantly different to the $\mathrm{SC}$ group. ${ }^{\dagger} p<.05$ significantly different to the $\mathrm{OC}$ group 

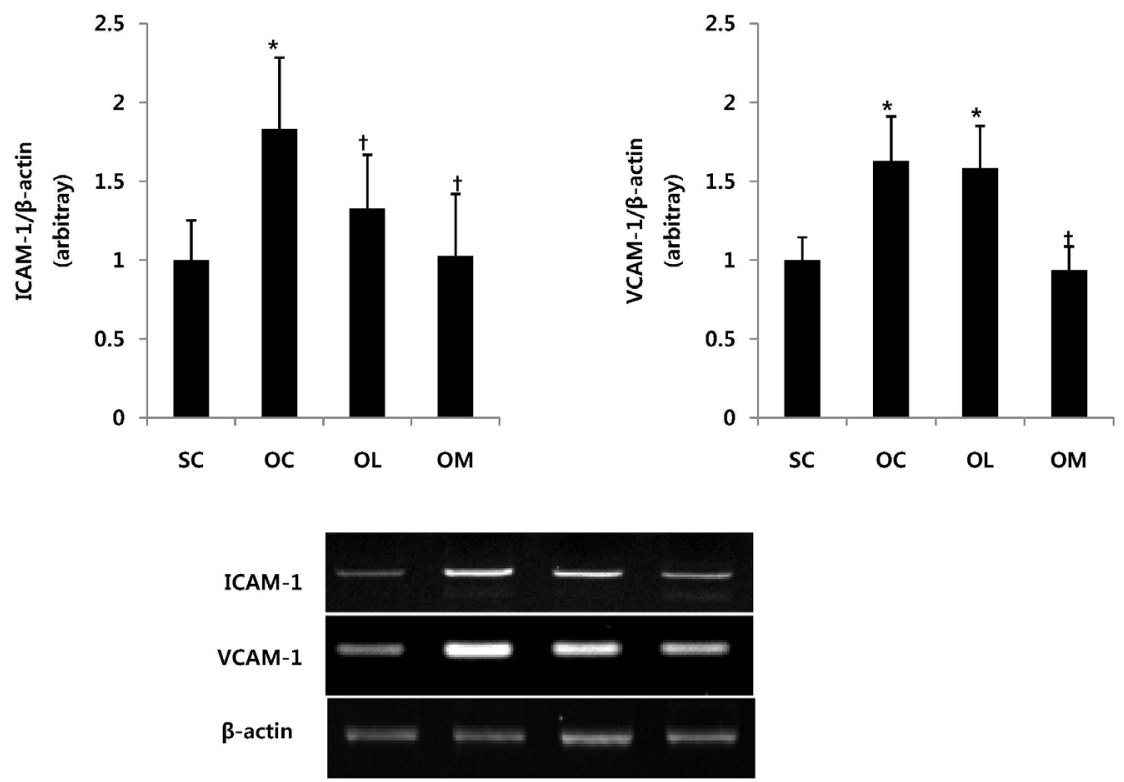

Fig. 2. Expression of ICAM-1 and VCAM-1 in ovariectomized rats. All values are expressed as means $\pm \mathrm{SD}$. $P$-values are the result of post hoc LSD test when the one-way ANOVA was significant. ${ }^{*} p<.05$ significantly different to the $\mathrm{SC}$ group. ${ }^{\dagger} p<.05$ significantly different to the OC group.

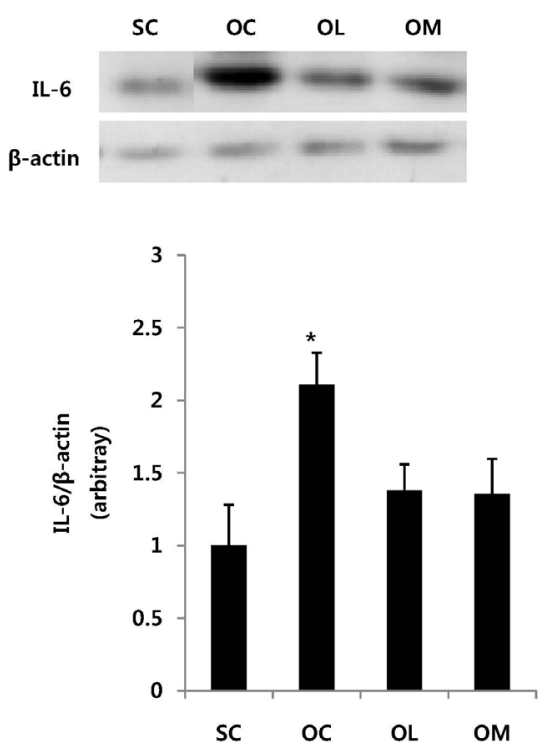

Fig. 3. Expression of IL-6, TLR4, TNF- $a$, MCP-1 in ovariectomized rats. All values are expressed as means \pm SD. $P$-values are the result of post hoc LSD test when the one-way ANOVA was significant. ${ }^{*} p<.05$ significantly different to the $\mathrm{SC}$ group. ${ }^{\dagger} p<.05$ significantly different to the OC group.

in comparison with OC group. The TLR4, TNF- $a$, and MCP-1 mRNA expression of OC group was significantly increased in comparison with $\mathrm{SC}$ group $(p<.01)$. After the exercise treatment of 16 weeks, OM group allowed to effectively reduce TLR4, TNF- $a$ and MCP-1 mRNA expression in comparison with OC group $(p<.01)$. However, OL group could not represent the significant difference. 


\section{DISCUSSION}

The ovariectomized animals have been used as an animal model to be improved obesity and metabolic imbalances that estrogen secretion appears by being limited [2,3]. In this study, the authors have investigated the effect of the low intensity exercise and moderate intensity exercise of 16 weeks on the morphological changes such as the body weight and adipose tissue weight by using the ovariectomized animal model, and attempted to utilize as a tool for the improvement of obesity by investigating the effect of each exercise treatment on the infiltration of macrophages and the regulation of inflammatory conditions in the visceral fat very relevant to the metabolic disease.

In this research result, the body weight and adipose tissue weight of the ovariectomized OVX group was significantly increased in comparison with the SC group without removing the ovary. However, after the exercise treatment of 16 weeks was completed, the body weight and adipose tissue weight showed the positive effect in the OM group that conducted the moderate intensity exercise in comparison with OC group, and the body weight showed the positive effect in OL group that conducted the low intensity exercise. These research results are consistent with the previous studies that the moderate intensity exercise allowed to effectively reduce the body weight and fat weight by increasing the energy consumption [18,19], and it is suggesting that the moderate intensity exercise can suppress the obesity induced in the menopause.

Recently, in the studies reported, the researchers have reported that the macrophage increased in adipose tissue is the risk factors aggravating inflammation situation $[9,10]$, and Kanda et al. [26] and Weisberg et al. [27] have reported that when the macrophage infiltration was blocked to the obese rats induced by the high fat dietary, the expression of inflammatory cytokines was significantly suppressed. In this study, the authors attempted to verify whether the low intensity exercise or the moderate intensity exercise can suppress the infiltration of F4/80 known as the marker of macrophage by using the ovariectomized animal model.

It could be confirmed that after the exercise treatment period of 16 weeks is completed, the infiltration of F480 in the ovariectomized OC group was significantly increased in comparison with SC group without removing the ovary. On the contrary to this, the OM group which is the moderate intensity exercise group effectively inhibited the infiltration of F480 compared to OC group. Because these results are also consistent with the result that Kawanishi et al. [28] have reported that the moderate intensity treadmill training had significantly reduced F480mRNA, it is considered that the results have suggested that the moderate intensity exercise has the function to positively suppress the infiltration of macrophages occurred due to a hormonal imbalance. It has been reported that the infiltration of macrophages in adipose tissue is controlled by the adipose tissue weight and the volume of lipid metabolism [29]. Thus, it is considered that the moderate intensity exercise used in this study effectively suppresses the infiltration of macrophages along with the reduction of body weight and adipose tissue by positively inducing the lipid metabolism in the adipose tissue of ovariectomized animal.

Moreover, the reason that there is the difference between the previous studies [2-7] and the results of this study is that cannot exclude the dietary effect because most previous studies using the ovariectomized animals had reported antiobesity effect when run parallel with the high fat dietary. However, it is the fact that in the ovariectomized animal model used in this study, the metabolic imbalance that appears due to fully decrease of estrogen was observed. In addition, in the previous studies, the authors have investigated the F480 known as a macrophage marker in mRNA level, but in this study, the researchers have verified the protein expression of F480, which is the result that the moderate intensity treadmill exercise demonstrated the sub-mechanism to effectively inhibit the macrophages infiltration along with the reduction of the adipose tissue of the ovariectomized animal.

However, the OL group that has performed the low intensity exercise did not effectively inhibit the expression of F480. In the case of menopausal women, the body weight and abdominal obesity are increased due to the reduction of energy consumption, and it has been reported that there was associated with the reduction of estrogen [30], and also it has suggested that the exercise intensity and period play a important role for the expression of estrogen receptor a while reporting that the moderate intensity exercise can increase the expression of estrogen receptor $a(E R a)$ in the liver of an ovariectomized animal [31]. Therefore, it is considered that in the case of the low intensity exercise used in this study, if the arbitration period was set to long-term, or the exercise that energy consumption was high was selected, the improvement effect in also the low intensity exercise group would be able to be obtained.

In the course of an inflammatory response, the cell adhesion molecules of largely ICAM-1, and VCAM-1 are being typically studied, and Bruun et al. [18] have suggested that moderate intensity exercise is a useful tool as the treatment method for the improvement of cardiovascular diseases by effectively reducing inflammatory cytokines and stricture molecules in 
the blood of cardiovascular patients. However, Davis et al. [32] have reported that ICAM-1 and E-selectin were significantly increased in vascular endothelial cells of the patients with coronary artery disease, but there is no significant difference for the expression of VCAM-1, and they have suggested that ICAM-1 is closely related to the movement and infiltration of each inflammatory cells. In this study, it was confirmed that the expression of ICAM-1 and VCAM-1 Mrna were significantly increased in OC group in comparison with SC group. In addition, the moderate intensity exercise of 16 weeks allowed to significantly inhibit the expression of ICAM-1과 VCAM-1 mRNA, and the low intensity exercise groups were not represented the difference between the ovariectomized control group. Because the expression of ICAM-1 and VCAM-1 appear differently depending on the environmental situation [33], it is considered that in future, the function research of the stricture molecule according to the exercise intensity should be attempted the studies applying various exercise treatment.

It has been known that the free fatty acid released in excessive in the blood induces the expression of the inflammatory cytokine of TNF- $a$ or IL- 6 by revitalizing the inflammatory receptors TLR4 in adipose tissue [33]. However, it has been reported that the exercise of moderate intensity plays a positive role also for the reduction of inflammatory cytokines along with the decrease of body weight and fat weight by enhancing the decomposition ability of fat metabolism [15-19].

In addition, it was confirmed that in the results of this study, the expression of TLR4, TNF- $a$ and MCP-1 in the adipose tissue of the ovariectomized control group was significantly increased in comparison with SC group. However, it was confirmed that the moderate intensity exercise has effectively suppressed the expression of inflammatory cytokine.

Recently, several studies have reported that the inflammation and metabolic diseases have improved in the adipose tissue of obese mice which inflammation receptor was broken [34-36]. These findings have reported that TNF- $a$ in the sub-mechanism of TLR4 in adipose tissue is the important regulators of the inflammatory gene expression, so the study on the treatment method that blocked the signal of TLR4 will be useful to improve and to prevent the metabolic diseases. Therefore, it is considered that the reduction of TNF-amRNA expression shown in this research result have been possibly controlled by significant inhibition of TLR4. But in this study, the concentration of free fatty acids known that activates TLR4, was not measured. In future research, if the positive change in the tissue along with the concentration change of lipid in serum is proved, it is judged that the importance of exercise will be established more firmly.

Taken together this research result, it was confirmed that the moderate intensity exercise of 16 weeks has positively improved the obesity induced in the ovariectomized animal model. In addition, it was identified that the moderate intensity exercise can control the activity reduction of inflammatory receptor and the expression of inflammatory genes by inhibiting the expression of the infiltration of macrophages and the stricture molecules in the visceral fat. However, the aerobic exercise of low intensity did not effectively improve the metabolic imbalance occurred by obesity. It is considered that in order to investigate the effect of low intensity exercise, in future, researchers should carefully consider the exercise period and frequency.

\section{ACKNOWLEDGEMENTS}

This work was supported by the National Research Foundation of Korea Grant funded by the Korean Government (NRF-2012S1A5A2A01019962).

All experiments were approved by the Animal Care and Use Committee at the Chungnam National University (CNU00203).

\section{REFERENCES}

[1] Bjrntorp P. The regulation of adipose tissue distribution in humans. Int J Obes Relat Metab Disord, 1996; 20: 291-302

[2] Rogers, NH, Perfield, JW, Strissel, KJ, Obin, MS, Greenberg, AS. Reduced energy expenditure and increased inflammation are early events in the development of ovariectomy-induced obesity. Endocrinology. 2009;150(5): 2161-2168

[3] Saengsirisuwan, V, Pongseeda, S, Prasannarong, M, Vichaiwong, K, Toskulkao, C. Modulation of insulin resistance in ovariectomized rats by endurance exercise training and estrogen replacement. Metabolism. 2009; 58(1):38-47.

[4] Garcia Rodriguez LA, Pfaff GM, Schumacher MC, Walker AM, Hoffmeister H. Replacement estrogen use and body weight index. Epidemiology. 1990;1:219-223.

[5] Geary N, Asarian L. Estradiol increases glucagon's satiating potency in ovariectomized rats. Am J Physiol Regul Integr Comp Physiol. 2001;281:R1290-R1294.

[6] Wade GN, Gray JM. Gonadal effects on food intake and adiposity: A metabolic hypothesis. Physiol Behav. 1979; 
22:583-593.

[7] Wing RR, Matthews KA. Weight gain at the time of menopause. Arch Intern Med, 1991;151:97-102.

[8] Jeong S, Han M, Lee H, Kim M, Kim J, Nicol CJ, Kim $\mathrm{BH}$, Choi JH, Oh GT, Yoon M. Effects of fenofibrate on high-fat diet induced body weight gain and adiposity in female C57BL/6J mice. Metabolism. 2004;53:12841289 .

[9] Tchernof A, Calles-Escandon J, Sites CK, Poehlman ET. Menopause,central body fatness, and insulin resistance: effects of hormone-replacement therapy. Coron Artery Dis. 1998;9:503-511.

[10] Nicole HR, James WP, Katherine JS, Martin SO, Andrew SG. Reduced Energy Expenditure and Increased Inflammation Are Early Events in the Development of Ovariectomy-Induced Obesity. Endocrine, 2009;150(5): 2161-2168.

[11] Weisberg SP, McCann D, Desai M, Rosenbaum M, Leibel RL, Ferrante AW Jr. Obesity is associated with macrophage accumulation in adipose tissue. J Clin Invest. 2003;112:1796-808.

[12] Lumeng CN, Bodzin JL, Saltiel AR. Obesity induces a phenotypic switch in adipose tissue macrophage polarization. J Clin Invest. 2007;117:175-84.

[13] Kang NSS, Pyo and Sohn EH. Inhibitory effects of allicin on TNF-a-induced ICAM-1 expression is associated with catalase. J. Korean Plant Res. 2009;22(6):552-557.

[14] Xu Y. and S. Li. Blockade of ICAM-1: a novel way of vasculitis treatment. Biochem. Biophys. Res. Commun. 2009;459-461.

[15] Sugiura H, Nishida H, Inaba R, Mirbod SM, Iwata H. Effects of different duration of exercise on macrophage function in mice. J. Appl. Physiol. 2001;90(3):789-794.

[16] Forner MA, Collazos ME, Barriga C De La Fuente, M, Rodriguez, AB, Ortega E. Effects of age on adherence and chemotaxis capacities of peritoneal macrophage. Influence of physical activity stress. Mech. Aging Dev. 1994;75:179-189.

[17] Ferrandez, MD, De la Fuente M. Effects of age, sex and physical exercise on the phagocytic process of murine peritoneal macrophages. Acta. Physiol. Scand. 1999; 166:47-53.

[18] Bruun, JM, Helge, JW, Richelsen, B, Stallknecht, B. Diet and exercise reduce low-grade infiammation and macrophage infiltration in adipose tissue but not in skeletal muscle in severely obese subjects. Am. J. Physiol. Endocrinol. Metab. 2006;290(5):E961-E967.

[19] Vieira, VJ, Valentine, RJ, Wilund, KR, Anto, N, Baynard, T, Woods, JA. Effects of exercise and low-fat diet on adipose tissue inflammation and metabolic complications in obese mice. Am. J. Physiol. Endocrinol. Metab. 2009;296(5):E1164-E1171.

[20] Garekani, E T, Mohebbi, H, Kraemer, RR, Fathi, R. Exercise training intensity/volume affects plasma and tissue adiponectin concentrations in the male rat. Peptides. 2011;32:1008-1012.

[21] Niaki AG, Farshidi Z, Fathi R. Effects of different endocrine training intensities on resting levels of skeletal muscle and liver glycogen concentrations in male rats. Int J Endocrinol Metab. 2010;8(2):79-81.

[22] Tjonna AE, Lee SJ, Rognmo O, et al., "Aerobic interval training versus continuous moderate exercise as a treatment for the metabolic syndrome: a pilot study", Circulation. 2008;118(4):346-354

[23] Kang SJ \& Kim BR. Effects of aerobic exercise intensity on insulin resistance, rennin-angiotensin II and C-reactive protein in patients with metabolic syndrome. Execise Science. 2009;18(4):443-454

[24] Drenth, JP, Krebber, R J, Bijzet and Van Der Meer, JW. lncreased circulating cytokine receptors and ex vivo interleukin-1 receptor antagonist and interleukin-1beta production but decreased tumour necrsis factor-alpha production after a 5-km run. EUR J. Clin. lnvest. 1998; 28:866 872

[25] Pedersen, B. K., Adam S., Peter, S. Exercise and interleukine-6. Curr Opin Hematol. 2001; 8: 137-141.

[26] Kanda H, Tateya S, Tamori Y, Kotani K, Hiasa K, Kitazawa R, Kitazawa S, Miyachi H, Maeda S, Egashira $\mathrm{K}$, Kasuga M. MCP-1 contributes to macrophage infiltration into adipose tissue, insulin resistance, and hepatic steatosis in obesity. J. Clin. Invest. 2006;116: 1494-1505.

[27] Weisberg SP, Hunter D, Huber R, Lemieux J, Slaymaker S, Vaddi K, Charo I, Leibel RL, Ferrante A. CCR2 modulates inflammatory and metabolic effects of high-fat feeding. J. Clin. Invest. 2006;116:115-124.

[28] Kawanish, N, Yano, H, Yokogawa, Y, Suzuki, K. Exercise training inhibits inflammation in adipose tissue via both suppression of macrophage infiltration and acceleration of phenotypic switching from M1 to M2 macrophages in high-fat-diet induced obese mice. Exerc Immunol Rev. 2010;16:105-118.

[29] Kosteli, A., et al., Weight loss and lipolysis promote a dynamic immune response in murine adipose tissue. J Clin Invest. 2010;120(10):3466-79.

[30] Asikainen T M, Kukkonen-Harjula K, Miilunpalo S. Exercise for health for early postmenopausal women: a systematic review of randomised controlled trials. Sports 
Med. 2004;34(11):753-778.

[31] Paquette, A, Wang, D, Gauthier, MS, Prud' homme D, Jankowski, M, Gutkowska, J, Lavoie JM. Specific adaptations of estrogen receptor alpha and beta transcripts in liver and heart after endurance training in rats. Mol Cell Biochem. 2007a;306(1-2):179-187.

[32] Davies, MJ, Gordon, JL, Gearing, AJ, Pigott, R, Woolf, $\mathrm{N}$, Katz, D. The expression of the adhesion molecules ICAM-1, VCAM-1, PECAM, and E-selectin in human atherosclerosis. J Pathol. 1993;171(3):223-229.

[33] Ursula Norman, M, Will, G, James, Michael, J, Hickey. Differential roles of ICAM-1 and VCAM-1 in leukocyte endothelial cell interactions in skin and brain of MRL/faslpr mice. 2008;84(1):68-76.
[34] Kim F, Pham M, Luttrell I, Bannerman DD, Tupper J, Thaler $\mathrm{J}$, et al. Toll-like receptor-4 mediates vascular inflammation and insulin resistance in diet-induced obesity. Circ Res. 2007;100:1589-96.

[35] Romanatto T, Roman EA, Arruda AP, Denis RG, Solon $\mathrm{C}$, Milanski $\mathrm{M}$, et al. Deletion of tumor necrosis factor-alpha receptor 1 (TNFR1) protects against dietinduced obesity by means of increased thermogenesis. J Biol Chem. 2009;284:36213-22.

[36] Poggi M, Engel D, Christ A, Beckers L, Wijnands E, Boon L, et al. CD40L deficiency ameliorates adipose tissue inflammation and metabolic manifestations of obesity in mice. Arterioscler Thromb Vasc Biol. 2011; 31:2251-60. 